\title{
Safety of Mebendazole Use During Lactation: A Case Series Report
}

\author{
Nour Karra ${ }^{1,2} \cdot$ Rana Cohen $^{1,2} \cdot$ Maya Berlin $^{2}$ Natalie Dinavitser ${ }^{2}$. \\ Gideon Koren $^{1,4} \cdot$ Matitiahu Berkovitch ${ }^{1,2,3}$
}

Published online: 13 September 2016

(c) The Author(s) 2016. This article is published with open access at Springerlink.com

\begin{abstract}
Introduction Mebendazole is an effective drug widely used in the treatment of parasitic infections. Although theoretically considered as safe during lactation, no studies have evaluated its potential adverse effects in infants of breastfeeding mothers.

Objectives We aimed to evaluate the safety of mebendazole in infants of lactating women treated with the drug.

Methods Women referred for consultation regarding mebendazole use were invited to participate in the study. Overall 45 lactating women treated with various protocols of mebendazole were recruited in this case series study.

Results Regardless of the treatment protocol used (single or repeated doses) mebendazole was well tolerated and was not associated with any adverse effects in infants of lactating mothers. There was mild GI irritability in two treated women.

Conclusion This study provides first evidence in humans as to the safety of mebendazole in breastfeeding.
\end{abstract}

Electronic supplementary material The online version of this article (doi:10.1007/s40268-016-0142-z) contains supplementary material, which is available to authorized users.

\footnotetext{
Nour Karra

nour_karra@yahoo.com

1 The Motherisk Program, Zerifin, Israel

2 Clinical Pharmacology Unit, Assaf Harofeh Medical Center, 70300 Zerifin, Israel

3 Affiliated to Sackler School of Medicine, Tel-Aviv University, Tel-Aviv, Israel

4 The Motherisk Program, Hospital for Sick Children and University of Toronto, Toronto, Canada
}

\section{Key points}

Mebendazole use during lactation is considered to be safe due to a low oral absorption and unlikely excretion in milk. However, there are no actual studies corroborating this assumption to date.

In this case series report, 45 breastfeeding mothers who were treated with mebendazole did not report any adverse reactions in their nursing infants. This study presents first evidence in humans as to this medication's safety during lactation.

\section{Introduction}

Medical professionals are often reluctant to prescribe pharmacological agents in the treatment of nursing mothers. Although recent statements by the American Academy of Pediatrics (AAP) support the safe use of numerous drugs during lactation $[1,2]$, concerns are often raised both by professionals and breastfeeding mothers as to the safety data of individual drugs. The lack of clinical data may often lead to unjustified avoidance of medications and, hence, suboptimal treatment of various maternal conditions. The issue of drug safety during lactation is particularly important as breastfeeding remains the gold standard for infant nutrition [3]. According to the World Health Organization (WHO), exclusive breastfeeding is recommended up to 6 months of age, with continued breastfeeding along with appropriate complementary foods up to two years of age or beyond [3]. 
In the present study we aimed to evaluate the safety of mebendazole in nursing mothers. Mebendazole (Vermox ${ }^{\circledR}$ ) is an effective anti-parasite drug used for the treatment of nematode infections in the GI tract. Mebendazole selectively damages cytoplasmic microtubules in the absorptive and intestinal cells of nematodes but not of the host. This irreversible microtubular damage leads to disruption of absorptive and secretory functions of the cells, which are essential to the worm's survival [4]. Inhibition of glucose uptake by the parasite leads to its starvation and death $[4,5]$.

Mebendazole is generally considered a well tolerated drug with few adverse effects [5, 6]. The most common side effects include abdominal pain and diarrhea which may occur transiently during therapy. This reaction may in part be a response to expulsion of the worms following therapy and can be more pronounced in cases of massive helminth infection [4]. Other adverse effects may include nausea and vomiting, elevated hepatic enzymes, neutropenia and rarely hepatitis, reported after long term mebendazole therapy, with higher than recommended doses [4].

Notably, the drug is poorly absorbed from the gut with $2-10 \%$ systemic bioavailability $[4,6]$. Thus, expected drug concentrations excreted in the milk of treated and breastfeeding mothers are negligible. Indeed, several isolated case reports reported undetectable milk levels of mebendazole following 3 days treatment with $200 \mathrm{mg}$ daily of the drug [6, 7]. The low oral and milk bioavailability renders the risks of exposure and side effects in the nursing infant very unlikely.

Nevertheless, there are no studies addressing the effects of mebendazole on infants of treated lactating mothers. This is despite high prescription rates of mebendazole in breastfeeding mothers. At the Drug Consultation Center (DCC) of Assaf Harofeh Medical Center a large number of consultations are related to anti-helmintic therapy in nursing mothers, providing an available pool of data on mebendazole.

\section{Methods}

The study was approved by the local ethics committee of Assaf Harofeh Medical Center. Women who called The DCC at Assaf Harofeh Medical Center to obtain information regarding potential risks of mebendazole to the nursing infant were invited to participate in the study and informed consent was obtained from all individual participants to subsequently use the collected data. A total of 58 women who called the DCC between June 2014 and January 2016 were included. All women had suffered from pinworm infection accompanied by disturbing rectal itching. Although baseline data were initially collected from 58 women who were all similar in age, birth outcome and prescribed mebendazole treatment protocol, thirteen women did not complete the follow up: 4 women eventually did not use the drug, while with the remaining 9 women communication was simply lost (non responders). Hence, a total of 45 breastfeeding mothers with mebendazole use and complete follow up were eventually enrolled (Table 1). Women receiving either a single dose or repeated doses of mebendazole were included (Table 2). Data were collected using a structured questionnaire (Supplementary data) and included the following information: maternal age, maternal medical history, the infant's gestational age and weight at birth, duration and dose of treatment, breastfeeding frequency per day and physical or behavioral changes in the nursing infant during treatment (rash, diarrhea, constipation, vomiting, fever, irritability or other). The adverse reactions were defined as changes in the condition of the infant and were observed and recorded by the mother (see detailed questionnaire in Supplementary data).

Table 1 Characteristics of mothers and infants exposed to mebendazole. Data are expressed as mean \pm SD

\begin{tabular}{ll}
\hline Parameter & $\begin{array}{l}\text { Mebendazole treatment } \\
\text { group }\end{array}$ \\
\hline Number of women & 45 \\
Maternal age (years) & $35.7 \pm 3.8$ \\
Birth week & $39.2 \pm 1.62$ \\
Birth weight (kg) & $3.28 \pm 0.5$ \\
Age at exposure (weeks) (range) & $16.37 \pm 19.5(1-114)$ \\
Age at follow up (weeks) (range) & $31.4 \pm 21.7(3-117)$ \\
\hline
\end{tabular}

Table 2 Treatment protocols used for mebendazole in the study group

\begin{tabular}{llc}
\hline Mebendazole treatment protocols used & \\
\hline $\begin{array}{l}\text { Daily dose } \\
(\mathrm{mg})\end{array}$ & Dosing regimen & $\begin{array}{c}\text { Sample } \\
\text { size }(N)\end{array}$ \\
\hline 100 & SD & 5 \\
100 & $1 \times 1 /$ day on day 1 & 22 \\
& Repeated after 7-14 days & 2 \\
100 & Once weekly for 3 weeks & 6 \\
100 & $1 \times 1 /$ day for 3 days & 4 \\
100 & $1 \times 1 /$ day for 3 days & 4 \\
200 & Additional SD after 10 days & \\
200 & $1 \times 2 /$ day for 3 days \\
& $1 \times 2$ repeated after 7-10 days & 1 \\
200 & Additional SD after 10 days & 1 \\
\hline
\end{tabular}

$S D$ single dose 


\section{Results}

Forty five women with mebendazole treatment during lactation were enrolled in this study. All variables of the study group are described in Table 1: maternal age, birth week, birth weight, age at exposure and age at follow up. Out of 45 nursing infants, $33(73 \%)$ were exclusively breast fed and in 12 infants breastfeeding was partial. The age of the exclusively breast fed infants at exposure time ranged between 1 and 30 weeks. Among the partially breast fed infants 8 were over the age of 20 weeks at exposure time to mebendazole. Out of 45 women, two treated women $(4.5 \%)$ reported mild GI irritability following mebendazole treatment which presented as flatulence or abdominal pain. In one breastfeeding mother in the study group milk production was reported to be slightly decreased during mebendazole treatment. Of note, these women were not different than the other 43 women in their baseline characteristics and had received one of the standard treatment regimens: the woman reporting flatulence was treated with a single dose while the woman reporting abdominal pain and decreased milk production was treated with a single dose repeated following 10 days. Mebendazole was well tolerated by the other 43 women (95.5\%), who did not have any adverse effects to report. No adverse reactions were recorded in any of the nursing infants.

\section{Discussion}

The current study provides the first human evidence on the safety of mebendazole treatment during lactation among 45 women who were retrospectively recruited. The overall results indicated no adverse reactions in 45 nursing infants. This supports the use of mebendazole to treat parasitic infections in lactating women, either as a single dose or in a repeated doses regimen when necessary. In view of the available pharmacokinetic data on the limited bioavailability of mebendazole mentioned earlier $[4,6]$, our reported observations are not surprising.

Mebendazole was reported to induce a slight decrease in milk production in one treated mother in our study. However, this phenomenon did not require the addition of supplemental artificial feeding to the infant nor did it require treatment discontinuation. This phenomenon was also reversible after treatment cessation (2-3 days following treatment completion) and probably should not be a reason for excessive concern or for treatment avoidance. It should also be emphasized that this reduction in milk production was subjectively reported by the mother, and no actual quantitative measurement of milk production was assessed. Therefore, although consistent with a previous report in the literature [7], it is somewhat difficult to base a solid causative link between this specific subjective report and mebendazole treatment. Furthermore, it is difficult to assess whether the GI irritability reported by 2 subjects in the study is attributed to the medication or to the parasite infestation.

In recent clinical guidelines published by the AAP, most medications were regarded as safe for breastfeeding mothers [1]. Only a small fraction of pharmacologic agents carry cautions in lactation due to proven adverse reactions on the infant. However, data on the excretion in milk and more importantly on the safety of a particular drug are not always available. Notably, when specific and solid information regarding drug safety in lactation is lacking, non experts may be more prone to be inappropriately influenced by available (though irrelevant) pregnancy-related drug data. For example mebendazole is not considered a proven teratogen [5]. However, according to the US FDA pregnancy classification it is still categorized as a Class $\mathrm{C}$ drug, due to embryotoxic or teratogenic effects in rats and insufficient safety data in humans [8]. Importantly, such pregnancy classification may negatively influence the general perception of the drug by medical practitioners and lead to over-estimated lactation risk. Consequently, mothers are often inappropriately advised to discontinue breastfeeding or avoid treatment with medications such as mebendazole because of un-based fears of adverse effects on their infants. This cautious approach may be unnecessary and even harmful in many cases [1]. In the case of infectious diseases untreated mothers may become a source of transfer of the infectious agent, putting their infants and other family members at risk. Furthermore, people often seek alternative, non-conventional treatments as they believe these are safer than the conventional "synthetic chemical" compounds [9]. Dietary supplements and herbal compounds undergo significantly less scrutiny by the FDA than conventional drugs. Such alternatives largely include "natural", herbal based and pseudo-homeopathic preparations which in many cases consist of a mixture of compounds, some of which are not even specified, in unknown concentrations and purity [10]. It should be emphasized that such preparations are often sold with no inspection or regulation of the appropriate authorities and medical professionals [10]. This treatment approach withholds great risks of un-useful treatment at best and fatal intoxications at worst.

The use of mebendazole in lactating women is quite common for the treatment of parasitic infections and is generally considered safe. An informal consultation group to the WHO concluded that a single oral dose of mebendazozle can be given to lactating women $[11,12]$. However, no systematic study has been yet performed to corroborate this assumption. Recognizing the need to corroborate the safe use of conventional medications, this study aimed to specifically evaluate the adverse effects of mebendazole in lactating mothers and their infants, and the results are indeed reassuring. 
Although encouraging, the study has several limitations that need to be acknowledged: first, the small sample size limits its statistic power. In addition, the use of a retrospective phone-based questionnaire may impose a recall bias. A prospective cohort, with an appropriate control group would have been a more powerful tool to corroborate our reported data. Yet, the present case series study is still the largest survey reported so far dealing with this previously neglected issue.

\section{Conclusion}

In this small case series report of 45 women, no adverse effects associated with mebendazole use during lactation were observed in the nursing infants.

\section{Compliance with Ethical Standards}

Conflict of interest N. Karra, R. Cohen, M. Berlin, N. Dinavitser, G. Koren and M. Berkovitch declare that they have no conflict of interest.

Funding No funding was received for this study.

Ethical approval The study was approved by the local ethics committee of Assaf Harofeh Medical Center and is in accordance with the 1964 Helsinki declaration and its later amendments.

Informed consent Informed consent was obtained from all individual participants to use the collected data.

Open Access This article is distributed under the terms of the Creative Commons Attribution-NonCommercial 4.0 International License (http://creativecommons.org/licenses/by-nc/4.0/), which permits any noncommercial use, distribution, and reproduction in any medium, provided you give appropriate credit to the original author(s) and the source, provide a link to the Creative Commons license, and indicate if changes were made.

\section{References}

1. Sachs HC. The transfer of drugs and therapeutics into human breast milk: an update on selected topics. From the American Academy of Pediatrics, clinical report. Pediatrics. 2013;132(3): e796-809.

2. American Academy of Pediatrics. The transfer of drugs and other chemicals into human milk. Pediatrics. 2001;108:776-89.

3. Global strategy for infant and young child feeding. World Health Organization, UNICEF, 2003. http://www.who.int/nutrition/ publications/infantfeeding/9241562218/en/. Accessed 15 April 2016.

4. Clinical Pharmacology website. http://www.clinicalpharmacology. com/Forms/drugoptions.aspx? cpnum $=365 \& \mathrm{n}=$ Mebendazole $\& \mathrm{t}=0$. Accessed 15 April 2016.

5. Schaefer C, Peters P, Miller RK. Drugs during pregnancy and lactation, treatment options and risk assessment. 3rd ed. Elsevier: Academic Press; 2015.

6. Hale TW, Rowe HE. Medications and mother's milk, a manual for lactational pharmacology. 16th ed. Plano: Hale Publishing, LP; 2014.

7. Rao TS. Does mebendazole inhibit lactation? NZ Med J. 1983;96(736):589-90.

8. Torp-Pedersen A, Jimenez-Solem E, Andersen JT, Broedbaek K, Torp-Pedersen C, and Poulsen HE. Exposure to mebendazole and pyrvinium during pregnancy: a Danish Nationwide Cohort Study. Infect Dis Obstet Gynecol. 2012:1-6. doi:10.1155/2012/769851.

9. Pallivalapila AR, Stewart D, Shetty A, Pande B, Singh R, McLay JS. Use of complementary and alternative medicines during the third trimester. Obstet Gynecol. 2015;125(1):204-11.

10. Cohen PA. American roulette-contaminated dietary supplements. NEJM. 2009;361(16):1523-5.

11. Allen HE, Crompton DW, de Silva N, et al. New policies for using anthelmintics in high risk groups. Trends Parasitol. 2002;18:381-2.

12. Report of The WHO Informal Consultation on The Use of Praziquantel During Pregnancy/lactation and Albendazole/ Mebendazole in Children Under 24 Months, Geneva 2002. World Health Organization Strategy Development and Monitoring for Parasitic Diseases and Vector Control (PVC) Control, Prevention and Eradication (CPE) Program on Communicable Diseases (CDS). 2002. http://www.who.int/schistosomiasis/resources/ resources_2002/en/. Accessed 15 April 2016. 